The Astrophysical Journal, 612:481-495, 2004 September 1

(C) 2004. The American Astronomical Society. All rights reserved. Printed in U.S.A.

\title{
LIMITS ON THE OPTICAL BRIGHTNESS OF THE $\epsilon$ ERIDANI DUST RING ${ }^{1}$
}

\author{
Charles R. ProffitT ${ }^{2}$ \\ Science Programs, Computer Sciences Corporation, 3700 San Martin Drive, Baltimore, MD 21218; proffitt@stsci.edu \\ Kailash Sahu, Mario Livio, John Krist, Daniela Calzetti, and Ron Gilliland \\ Space Telescope Science Institute, Baltimore, MD 21218 \\ Carol Grady ${ }^{3}$ \\ NOAO/STIS, Code 681, NASA Goddard Space Flight Center, Greenbelt, MD 20771 \\ DON LINDLER \\ Sigma Research and Engineering, Lanham, MD 20706 \\ Bruce Woodgate, ${ }^{4}$ Sara Heap, ${ }^{4}$ Mark Clampin, and Theodore R. Gull ${ }^{4}$ \\ Laboratory for Astronomy and Solar Physics, Code 681, NASA Goddard Space Flight Center, Greenbelt, MD 20771 \\ AND \\ CASEY M. Lisse \\ Department of Astronomy, University of Maryland, College Park, MD 20742 \\ Received 2004 March 2; accepted 2004 May 13
}

\begin{abstract}
The STIS CCD camera on the Hubble Space Telescope (HST) was used to take deep optical images near the $\mathrm{K} 2 \mathrm{~V}$ main-sequence star $\epsilon$ Eridani in an attempt to find an optical counterpart of the dust ring previously imaged by submillimeter observations. Upper limits for the optical brightness of the dust ring are determined and discussed in the context of the scattered starlight expected from plausible dust models. We find that even if the dust is smoothly distributed in symmetrical rings, the optical surface brightness of the dust, as measured with the HST STIS CCD clear aperture at $55 \mathrm{AU}$ from the star, cannot be brighter than about $25 \mathrm{STMAG} \mathrm{arcsec}^{-2}$. This upper limit excludes some solid-grain models for the dust ring that can fit the IR and submillimeter data. Magnitudes and positions for $\approx 59$ discrete objects between 12 ."5 and 58 from $\epsilon$ Eri are reported. Most if not all of these objects are likely to be background stars and galaxies.
\end{abstract}

Subject headings: circumstellar matter — stars: individual ( $\epsilon$ Eridani)

\section{INTRODUCTION}

A substantial fraction $(\approx 15 \%)$ of main-sequence stars show evidence of excess IR or submillimeter flux due to thermal emission from dust located at distances of $30 \mathrm{AU}$ or more from the stars; i.e., locations comparable to that of the Kuiper Belt in our own solar system. This was first discovered using IRAS observations (e.g., Aumann et al. 1984; Aumann 1985; Gillett \& Aumann 1983), and subsequent observations with the James Clarke Maxwell Telescope's Submillimeter Common-User Bolometer Array (JCMT SCUBA) have directly imaged the dust distribution in a few of these systems (Holland et al. 1998), including $\epsilon$ Eri (Greaves et al. 1998).

$\epsilon$ Eridani is a $\mathrm{K} 2 \mathrm{~V}$ main-sequence star at a distance of about 3.2 pc. It is believed to be a relatively young system (<1 Gyr; Song et al. 2000; Soderblom \& Dappen 1989), with a mass slightly less than our own Sun. From radial velocity measurements, Hatzes et al. (2000) have reported evidence for

\footnotetext{
${ }^{1}$ Based on observations made with the NASA/ESA Hubble Space Telescope, obtained at the Space Telescope Science Institute, which is operated by the Association of Universities for Research in Astronomy, Inc., under NASA contract NAS 5-26555. These observations are associated with proposal GO-09037.

${ }^{2}$ Also at: Space Telescope Science Institute, and Institute for Astrophysics and Computational Science at the Catholic University of America.

3 Also at: Eureka Scientific, 2452 Delmer Street, Suite 100, Oakland, CA 94602-3017.

${ }^{4}$ Member of Space Telescope Imaging Spectrograph Investigation Definition Team.
}

a planet in this system with a semimajor axis of $3.4 \mathrm{AU}$ and $m \sin i=0.86 M_{\mathrm{J}}$.

The $850 \mu \mathrm{m}$ observations of Greaves et al. (1998) show a ringlike structure around $\epsilon$ Eri. The maximum surface brightness of this ring is located at a radius of $\approx 17^{\prime \prime}(55 \mathrm{AU})$ from the star, with some flux extending out as far as $36^{\prime \prime}$ (115 AU). The observed $850 \mu \mathrm{m}$ flux shows the ring to be asymmetrical, with several bright clumps. It has been suggested that structures of this kind can be caused by resonant interactions of dust with planets in or near the ring (Liou \& Zook 1999; Ozernoy et al. 2000; Quillen \& Thorndike 2002).

In an attempt to detect an optical counterpart of this ring, we undertook observations with the Hubble Space Telescope's Space Telescope Imaging Spectrograph (HST STIS) of $\epsilon$ Eri, using this instrument's CCD camera, as part of HST GO program 9037 (PI: Mario Livio). A full description of the STIS instrument can be found in Kim Quijano et al. (2003).

While this camera can be used with a number of coronagraphic wedges, saturation of the detector by the wings of the stellar point-spread function (PSF) near the edges of the wedge would severely limit the exposure time achievable in a single image. Therefore, we instead used the $52^{\prime \prime} \times 52^{\prime \prime}$ clear CCD aperture and placed the star $\approx 5^{\prime \prime}$ off the edge of the detector. The K0 IV star $\delta$ Eri was also observed as a PSF comparison star.

\subsection{Details of Previous Observations}

Gillett (1986) reconsidered the IRAS observations of $\epsilon$ Eri and concluded that the intrinsic FWHM of the source flux at 
TABLE 1

IR and Submillimeter Measurements of Dust around $\epsilon$ Eri

\begin{tabular}{|c|c|c|c|}
\hline $\begin{array}{l}\text { Wavelength } \\
\qquad(\mu \mathrm{m})\end{array}$ & $\begin{array}{c}\text { Dust Flux } \\
(\mathrm{mJy})\end{array}$ & Source & Comments \\
\hline $1200 .$. & $21.4 \pm 5.1$ & Schütz et al. (2004) & \\
\hline $850 \ldots \ldots$ & $40 \pm 3$ & Greaves et al. (1998) & \\
\hline 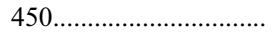 & $185 \pm 103$ & Greaves et al. (1998) & \\
\hline ..................... & 1780 & $I R A S$ & Photospheric flux subtracted \\
\hline 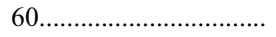 & 1340 & $I R A S$ & Photospheric flux subtracted \\
\hline 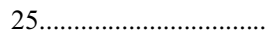 & 270 & $\operatorname{IRAS}$ & Photospheric flux subtracted \\
\hline
\end{tabular}

$60 \mu \mathrm{m}$ was less than $17^{\prime \prime}$ in the IRAS scan direction and less than $11^{\prime \prime}$ in the perpendicular direction. If this suggestion is correct, it would imply that the bulk of the IRAS emission comes from a region inside of the ring detected by Greaves et al. (1998). However, both the submillimeter ring and the IRAS size limits suggested by Gillett are significantly smaller than the nominal IRAS $60 \mu \mathrm{m}$ resolution at of about $1^{\prime}$ (Beichman et al. 1985), and therefore Gillett's conclusions should be treated cautiously.

Greaves et al. (1998) observed the system with SCUBA at both 850 and $450 \mu \mathrm{m}$. The $850 \mu \mathrm{m}$ observations used a beam of $15^{\prime \prime}$ FWHM, and Greaves et al. published smoothed versions of the images produced by these observations. The $\mathrm{S} / \mathrm{N}$ of the $450 \mu \mathrm{m}$ observations is too low to give any useful spatial information (no image was published), but it does supply a useful measure of the total flux.

Schütz et al. (2004) obtained $1200 \mu \mathrm{m}$ measurements using a $25^{\prime \prime}$ beam, which are consistent with Greave et al.'s measurements. Several previous submillimeter observations (Chini et al. 1990, 1991; Zuckerman \& Becklin 1993) at various wavelengths $(800-1300 \mu \mathrm{m})$ had used single pointings with beam sizes and background chopping too small to properly measure the structure detected by Greaves et al. (see also Weintraub \& Stern 1994). While these observations may provide some useful constraints, they cannot be used as direct measures of the flux and are not further considered here.

We summarize the IRAS, Greaves et al. (1998), and Schütz et al. (2004) data in Table 1. In this table we adopt Greaves et al's color corrections, as well as their corrections for the stellar contribution to the total flux, and present only the flux attributed to the dust alone.

At $55 \mathrm{AU}$, the IRAS and SCUBA data imply grain temperatures of about $30 \mathrm{~K}$, close to the equilibrium blackbody temperature. The very flat $450 \mu \mathrm{m}$ to $850 \mu \mathrm{m}$ flux ratio (4.6士 2.6) requires the presence of large grains $(>100 \mu \mathrm{m})$, which can emit efficiently at submillimeter wavelengths. At this distance, the timescale for the orbital decay of $100 \mu \mathrm{m}$ grains due to the
Poynting-Robertson effect is about $7 \times 10^{8} \mathrm{yr}$, a timescale that is comparable to the inferred age of the system. This would seem to argue against a substantial population of smaller grains in the outer parts of the system. However, recent works on various debris disk systems (e.g., Wyatt et al. 1999; Li et al. 2003) have shown that this kind of interpretation is overly simplistic. The submillimeter emissivity of grains drops rapidly enough with decreasing grain size that a substantial population of smaller grains has little effect on the submillimeter flux ratios, and the collision and fragmentation rate of the large grains required by the submillimeter data is still high enough to replenish the smaller grains faster than the Poynting-Robertson effect can remove them. We will see that the nature and abundance of these smaller grains has dramatic effects on the optical detectability of the dust in the $\epsilon$ Eri system.

\section{DESCRIPTION OF THE OBSERVATIONS}

The HST observations in this program were made on 2002 January 26, using six adjacent single-orbit visits (see Table 2). In each visit, after taking an ACQ exposure to determine the position of the targeted star, 15 offset exposures of $109 \mathrm{~s}$ each were taken using the unfiltered STIS CCD in imaging mode. During these offset exposures the bright star was located about $5^{\prime \prime}$ off the detector (near CCD pixel coordinates $x=1127, y=$ 514). The first and last orbits were used to observe the PSF of the comparison star, $\delta$ Eri, at two different orientations differing by $30^{\circ}$. The four intermediate orbits were used to observe the primary target at four different orientations separated by $10^{\circ}$ intervals. In each of these latter exposures, the position of the brightest submillimeter clump observed by Greaves et al. (1998) was imaged on the $1024 \times 1024$ pixel detector.

The clear aperture used with the STIS CCD is designated as the "50CCD" aperture in STIS documentation. It has a field of view of almost $52^{\prime \prime} \times 52^{\prime \prime}$, and has a very broad bandpass, with significant throughput from about 2000 to $10200 \AA$. Prior to the installation of the Advanced Camera for Surveys

TABLE 2

Deep Offset 50CCD Observations

\begin{tabular}{|c|c|c|c|c|c|}
\hline Target & Data Set & R.A. & Decl. & $\begin{array}{l}y \text {-axis Orientation } \\
(\text { deg. E of } \mathrm{N})\end{array}$ & $\begin{array}{c}\text { Expected Clump } \\
\text { Location } \\
\text { (pixels) }\end{array}$ \\
\hline$\delta$ Eri OFF1 .................. & o6eo01020 & $0343 \quad 16.95$ & -094552.09 & 10.0544 & \\
\hline$\epsilon$ Eri OFF1 & o6eo02020 & 033257.75 & -092735.20 & 10.0546 & 735,446 \\
\hline$\epsilon$ Eri OFF2 ................ & o6eo03020 & 033257.66 & -092740.42 & 20.0547 & 729,515 \\
\hline$\epsilon$ Eri OFF3 .................. & o6eo04020 & 033257.50 & -092745.31 & 30.0548 & 734,584 \\
\hline$\epsilon$ Eri OFF4 .................. & o6eo05020 & 033257.29 & -092749.74 & 40.0550 & 752,651 \\
\hline$\delta$ Eri OFF4 & o6eo06020 & 034316.49 & -094606.64 & 40.0548 & $\ldots$ \\
\hline
\end{tabular}


TABLE 3

F25ND3 OBSERVATIONS

\begin{tabular}{|c|c|c|}
\hline Target & Data Set & $\begin{array}{c}\text { Exposure Time } \\
\text { (s) }\end{array}$ \\
\hline$\delta$ Eri............................... & o6eo01010 & 0.4 \\
\hline$\epsilon$ Eri................................ & o6eo02010 & 0.6 \\
\hline 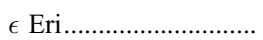 & o6eo03010 & 0.6 \\
\hline 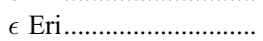 & o6eo04010 & 0.4 \\
\hline 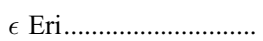 & o6eo05010 & 0.4 \\
\hline$\delta$ Eri............................. & o6eo06010 & 0.6 \\
\hline
\end{tabular}

(ACS), STIS 50CCD observations provided the most sensitive HST mode for deep imaging. We will, for the most part, give our observed STIS magnitudes in STMAG units, where the magnitude is defined as $-2.5 \log \left(F_{\lambda}\right)-21.10$, with $F_{\lambda}$ in ergs s${ }^{-1} \mathrm{~cm}^{-2} \AA^{-1}$. For STIS 50CCD imaging, the conversion to a magnitude system that uses Vega as the zero point is VEGAMAG $(50 \mathrm{CCD})=\mathrm{STMAG}(50 \mathrm{CCD})-0.36$. In addition, at the beginning of each orbit a very short observation was taken of each star using the STIS CCD with the F25ND3 filter (Table 3).

\section{DATA ANALYSIS}

\subsection{Basic Analysis}

The standard STIS pipeline software was used to produce bias- and dark-subtracted and flat-fielded images of individual subexposures (flt files). Comparison with median filtered images was done to identify and correct hot pixels that were not handled properly in the standard analysis; about $0.5 \%$ of pixels were corrected in this way.

Each of the 15 subexposure flt files was rearranged into five separate files, each containing three adjacent subexposures, and these files were input into the STSDAS STIS routine ocrreject to produce five separate cosmic-ray-rejected (crj) files for each of the six visits: $30 \mathrm{crj}$ files in total.

In each of these crj files, the two diffraction spikes from the star $\left(\epsilon\right.$ Eri or $\delta$ Eri) located $5^{\prime \prime}$ off the edge of the detector are the brightest features visible. These were used to register the images. As we are primarily interested in the relative offsets between the images, any small systematic offset from the real location of the star relative to the detector is unimportant. Each of the $30 \mathrm{crj}$ files was then shifted so as to put the intersection of the diffraction spikes at the mean location measured for all visits. The shifts applied were up to \pm 0.7 pixels in $x$ and \pm 0.3 pixels in $y$. Because we wanted to distort the high $\mathrm{S} / \mathrm{N}$ pattern of the PSF as little as possible, we shifted the images using a seven-point sinc interpolation function with the IRAF imshift routine. The shifted crj files for each visit were then combined by again using the STIS ocrreject routine, producing a single aligned crj file for each visit.

We investigated whether there was any advantage in shifting each individual subexposure, rather than combining them first by groups of three before shifting and coadding, but this does not appear to significantly improve the final co-added image.

\subsection{PSF Subtraction}

Subtraction of the PSF from the wings of the a bright star is very sensitive to small mismatches in the target and PSF star's spectral energy distribution, as well as to small changes in telescope focus and breathing (i.e., changes in image quality caused by flexure of HST and STIS optical elements). We use two separate techniques to subtract $\epsilon$ Eri's PSF. Rolldeconvolution techniques use the target as its own PSF star, by taking back-to-back observations at different orientations. Direct subtraction of the $\epsilon$ Eri and $\delta$ Eri observations is also done. A comparison of different PSF subtraction techniques for STIS coronagraphic observations was done by Grady et al. (2003), and much of their discussion is also relevant here.

\subsubsection{Roll Deconvolution of $\epsilon$ Eri}

The goal of the roll deconvolution is to use images taken at different orientations to separate the real sky image from the PSF of the bright nearby star. This technique eliminates any problem with mismatches in the shape of the PSF due to differences in the spectral energy distributions, but it has the disadvantage that any circularly symmetric features or arclike structures larger than the change in roll angle will be included with the PSF rather than as part of the sky image. Unfortunately, these are exactly the type of structures most likely in a circumstellar debris disk.

To obtain a first approximation to the PSF of $\epsilon$ Eri, we combined the four aligned and co-added $\epsilon$ Eri crj images, rejecting points that were high or low by more than $3 \sigma$ from the median value for that pixel location by using the IRAF imcombine routine with the ccdclip algorithm. As the images were not yet rotated to align them on the sky, this clips out real objects as if they were cosmic rays. This trial PSF was then subtracted from each of the original images. These subtracted images were each rotated about the position of $\epsilon$ Eri to align the positions on the sky and then combined by taking a straight average of the values at each pixel, but with locations near the main diffraction spikes or the obstructed edges of the 50CCD aperture masked out of the average. We found that masking out a rather wide strip (about 3"4 in the diagonal direction) around the main diffraction spikes gave the best results.

A number of faint objects are clearly visible in the field of view (see Fig.1). A mask was created for each unrotated image that identifies pixels that are affected by real objects on the sky. A final PSF for $\epsilon$ Eri was then made in the same way as the initial PSF image, but with these sky objects masked out before taking the average. The new PSF was then subtracted from the shifted crj images, and the subtracted files were again rotated into alignment and averaged after masking out the diffraction spikes and aperture edges.

To summarize, we masked out the sky objects when averaging the unrotated and unsubtracted images to create the PSF, and then masked out the diffraction spikes and aperture edges when averaging the rotated and PSF-subtracted images to create the image of the sky. In principle, this procedure could be iterated to refine the separation between the sky and PSF images, but we simply use the second version of the sky and PSF images produced by this procedure.

\subsubsection{Direct Subtraction of $\epsilon$ and $\delta$ Eri PSFs}

\subsubsection{1. $\delta$ Eri PSF}

The procedure used to produce the $\delta$ Eri PSF was similar to that used to produce the $\epsilon$ Eri PSF. It was necessary to mask out a generous region around the brightest background star in each of the two $\delta$ Eri images to avoid introducing obvious artifacts in the subtraction.

\subsubsection{Relative Normalization of the Two Stars}

Before subtracting the $\delta$ Eri PSF from the observations of $\epsilon$ Eri, it is necessary to know both $r$, the relative normalization 


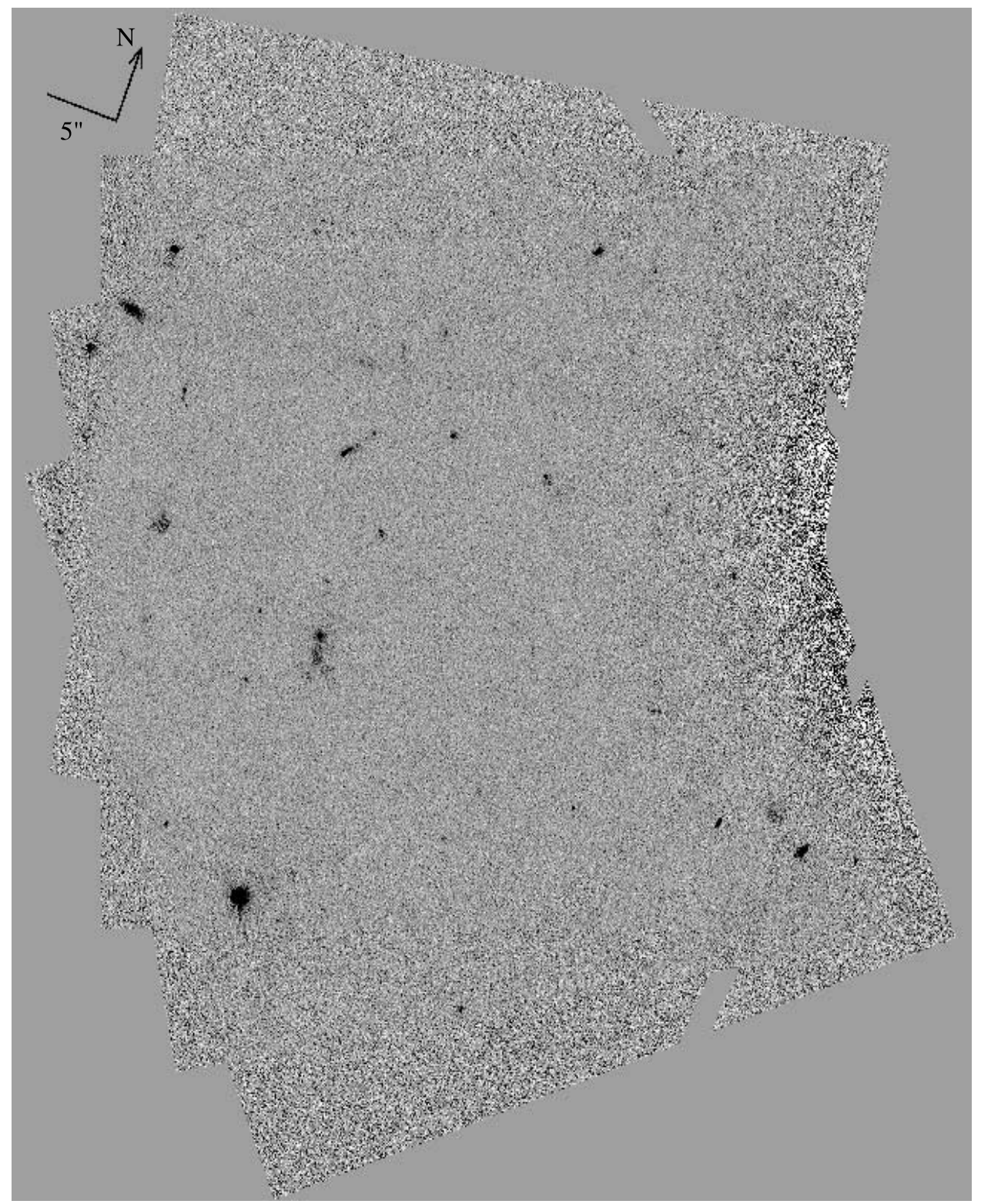

Fig. 1.-Full mosaicked, roll-subtracted image of the targeted field near $\epsilon$ Eri. This, and other images of this field, are aligned with the STIS observation o6eo03020, with the $+y$ direction aligned $20^{\circ}$ east of north. The compass drawn in the upper left corner of this figure is $5^{\prime \prime}$ on a side.

of the two PSFs, and $b$, the sky background level. The PSFsubtracted images will then be calculated as $I=\left(I_{\epsilon}-b\right)-$ $r\left(P_{\delta}-b\right)$.

To calculate the relative normalization, we need to consider the spectral energy distributions (SEDs) of the two stars. We approximate these SEDs by using the broadband photometry (Table 4) taken from the Lausanne online database (Mermilliod et al. 1997). Longward of the $V$ band, the two stars have very similar spectral distributions, but $\epsilon$ Eri is a bit bluer at shorter wavelengths. We supplement this photometry with $I U E$ data for shorter wavelengths to provide a rough SED for each star (Table 5), which is then used with SYNPHOT to predict STIS CCD imaging magnitudes in the 50CCD and F25ND3 filters (Table 6). The predicted F25ND3 magnitudes are about 8\% brighter than observed, but the predicted ratio of the two stars' F25ND3 count rates matches the observed ratio to within $0.2 \%$. This gives us a fair measure of confidence that the predicted flux ratio for STIS CCD imaging with the unfiltered 50CCD aperture will also be correct.
We have also constructed Tiny Tim (Krist 1993, 1995) models using the above SEDs as input. Tiny Tim only calculates on-axis PSFs for STIS, and does not calculate the PSF beyond a 4".5 radius. The ratio of the Tiny Tim PSFs does show that, when normalized to the same total flux, the slightly bluer $\epsilon$ Eri SED results in a PSF at 4."5 that is about $0.4 \%$

TABLE 4

Photometry of $\epsilon$ Eri And $\delta$ Eri

\begin{tabular}{|c|c|c|}
\hline Band & $\epsilon$ Eri & $\delta$ Eri \\
\hline$V \ldots$ & 3.726 & 3.527 \\
\hline$B-V \ldots$ & 0.882 & 0.922 \\
\hline$U-B \ldots \ldots \ldots$ & 0.584 & 0.686 \\
\hline$V-R_{\mathrm{C}} \ldots \ldots \ldots \ldots$ & 0.504 & 0.505 \\
\hline 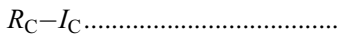 & 0.440 & 0.434 \\
\hline$V_{\mathrm{C}}-I_{\mathrm{C}} \ldots \ldots \ldots \ldots \ldots \ldots \ldots \ldots \ldots \ldots \ldots \ldots \ldots \ldots \ldots \ldots$ & 0.940 & 0.939 \\
\hline .................... & 0.55 & 0.56 \\
\hline
\end{tabular}


TABLE 5

Adopted Flux Distributions for $\epsilon$ Eri and $\delta$ Eri for PSF Calculations

\begin{tabular}{|c|c|c|c|}
\hline $\begin{array}{c}\lambda \\
(\AA)\end{array}$ & $\epsilon$ Eri & $\delta$ Eri & Ratio \\
\hline $2700 \ldots \ldots \ldots \ldots \ldots \ldots \ldots$ & $3.67 \times 10^{-12}$ & $2.75 \times 10^{-12}$ & 1.335 \\
\hline $3000 \ldots \ldots \ldots \ldots \ldots \ldots \ldots$ & $1.15 \times 10^{-11}$ & $1.11 \times 10^{-11}$ & 1.036 \\
\hline 3646.235 ................... & $3.63 \times 10^{-11}$ & $3.82 \times 10^{-11}$ & 0.950 \\
\hline $4433.491 \ldots \ldots \ldots \ldots \ldots \ldots . . .$. & $8.74 \times 10^{-11}$ & $1.01 \times 10^{-10}$ & 0.865 \\
\hline 5492.883.......................... & $1.14 \times 10^{-10}$ & $1.37 \times 10^{-10}$ & 0.832 \\
\hline 6526.661_...................... & $1.08 \times 10^{-10}$ & $1.30 \times 10^{-10}$ & 0.831 \\
\hline 7891.114 ….................. & $8.92 \times 10^{-11}$ & $1.07 \times 10^{-10}$ & 0.833 \\
\hline $12347.43 \ldots \ldots \ldots \ldots \ldots \ldots$ & $4.02 \times 10^{-11}$ & $5.02 \times 10^{-11}$ & 0.801 \\
\hline 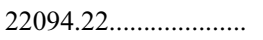 & $8.38 \times 10^{-12}$ & $1.06 \times 10^{-11}$ & 0.791 \\
\hline
\end{tabular}

lower than that of $\delta$ Eri. Taking the predicted 50CCD ratio of 0.837 , and then assuming the same difference between the predicted and observed ratios as was found for the direct F25ND3 images $(0.837-0.845)$, would give an expected ratio of 0.839 . Extrapolating the radial color difference found in the Tiny Tim models, we estimate an additional correction of about -0.005 near $17^{\prime \prime}$, which yields an estimated normalization ratio of 0.834 . If this ratio is adopted, it is also necessary to assume a mean sky background of $\approx 0.077 e^{-}$ pixel $^{-1} \mathrm{~s}^{-1}$ if the farthest parts of the two PSFs are to match. This is comparable to the expected sky brightness.

The results of this subtraction are shown in Figure 2. There appears to be substantial excess light near $\epsilon$ Eri, amounting to about $1 \%$ of the unsubtracted PSF at the same radius, and a number of concentric rings are visible. To illustrate this diffuse structure, we took the difference between the $\epsilon$ and $\delta$ Eri PSFs (with the sky objects removed), and measured the mean surface brightness as a function of radius (Figs. 3 and 4). We can minimize the central halo around $\epsilon$ Eri by increasing the normalization constant to about 0.842 (see Fig. 5), but the concentric rings remain, with a mean surface brightness of 25.5 to 25 STMAG $\operatorname{arcsec}^{-2}$. If we assume that the rings extend all the way around the star, this flatter normalization corresponds to a total integrated STMAG of 17.4 for the rings.

This structure seems suspiciously symmetrical to be consistent with the observed clumpiness in the submillimeter observations, but the radial distribution of the flux, with a broad hump between 15 and $30^{\prime \prime}$, is roughly consistent with the radial dependence of the $850 \mu \mathrm{m}$ flux as shown in Figure 2 of Greaves et al. (1998). The rings are broader than PSF subtraction artifacts previously seen in STIS coronagraphic imaging (Grady et al. 2003); however, few images expose the far PSF wings this deeply. One example of ACS coronagraphic observations of Arcturus did show that breathing-induced changes between visits can lead to a ring in the subtracted image with a radius of $13^{\prime \prime}$ and a surface brightness $20.5 \mathrm{mag}$ $\operatorname{arcsec}^{-2}$ fainter than the star (see Fig. 5.12 of Pavlovsky et al. 2003). It may be that the rings in our STIS data are a similar artifact. However, we do not completely ignore the possibility

TABLE 6

STIS CCD Imaging Photometry

\begin{tabular}{cccc}
\hline \hline Filter & $\epsilon$ Eri & $\delta$ Eri & Flux Ratio $(\epsilon / \delta)$ \\
\hline 50CCD (predicted) ................. & 4.023 & 3.830 & 0.8371 \\
F25ND3 (predicted) ................. & 4.038 & 3.842 & 0.8345 \\
F25ND3 (observed) ............... & 3.956 & 3.762 & 0.837 \\
\hline
\end{tabular}

that the rings might be real, and consider whether they could be plausibly modeled by dust in the $\epsilon$ Eri system.

\section{RESULTS}

\subsection{Comparison of the STIS Images with the Submillimeter Emission}

\subsubsection{Comparison with Roll-subtracted Images}

Figure 6 shows the roll-subtracted data from part of Figure 1, but at 2 times the scale of that figure and after smoothing with a $5 \times 5$ boxcar filter to suppress the smallscale noise. Overlaid on this figure is the submillimeter contour map of Greaves et al. (1998), positioned assuming that the submillimeter emission shares the proper motion of $\epsilon$ Eri. The inner contour level of the brightest submillimeter peak is about $5^{\prime \prime}$ in diameter.

There are a substantial number of faint objects detected in the region of the submillimeter emission, but the density of such objects is not appreciably greater than elsewhere in the image. There is no apparent correlation between these objects and the submillimeter flux, and most or all of these objects are probably background galaxies or stars. Note that the orbital period for material in the dust ring is about $500 \mathrm{yr}$, and the expected orbital motion is only about $0.2 \mathrm{yr}^{-1}$.

Figure 7 shows the same data, but leaves the submillimeter contours where they were actually observed in 1997-1998; i.e., they are not corrected for the $\approx 4^{\prime \prime}$ that $\epsilon$ Eri moved during the intervening years. In this figure, the brightest submillimeter clump is just outside the $2^{\prime \prime}$ submillimeter pointing uncertainty of what appears to be a background galaxy with STMAG 24.7 (this brightness includes all the clumps in this extended object).

Figure 8 compares the proper-motion-corrected submillimeter contours with a $5 \times 5$ boxcar smoothing of the image that results when subtracting the $\delta$ Eri PSF from the $\epsilon$ Eri data. One of the bright rings is roughly at the location of the submillimeter emission, but there is no correlation with the bright knots.

\subsection{Detected Objects near $\epsilon$ Eri}

Nearly 60 distinct objects are visible in the field of view, including about half a dozen within $20^{\prime \prime}$ of $\epsilon$ Eri. Most of these objects appear to be slightly extended, and many have complex morphologies. There is no apparent increase in their density at close distances to $\epsilon$ Eri, and most are probably background galaxies. Two relatively bright point sources are visible at distances of 51".5 (STMAG 19.6) and near 54" (STMAG 22.2). A list of detected objects, their locations, and their magnitudes is given in the Appendix (Table 9). For comparison, from the magnitudes and colors given by Chabrier et al. (2000) for models of 1 Gyr old brown dwarfs with dusty atmospheres, we estimate that a $0.05 M_{\odot}$ brown dwarf in the $\epsilon$ Eri system would have a STIS 50CCD STMAG of $\sim 19$, and a $0.03 M_{\odot}$ brown dwarf an STMAG of $\sim 27.5$.

\subsection{Point Source and Surface Brightness Detection Limits}

We measured the pixel-to-pixel rms variation in apparently blank regions of the final composite roll-subtracted image as a function of radius from $\epsilon$ Eri. The point-to-point rms noise varies smoothly and can be fitted as $\sigma \approx 31 / r^{3.16}+0.011 e^{-}$ $\mathrm{s}^{-1}$, where $r$ is the distance in arcseconds from $\epsilon$ Eri. The measured noise is close to that predicted by a simple noise model based on the read noise and the total number of counts in the PSF as a function of radius. Within $30^{\prime \prime}$ of the star, the noise in the PSF-subtracted image is dominated by the Poisson 


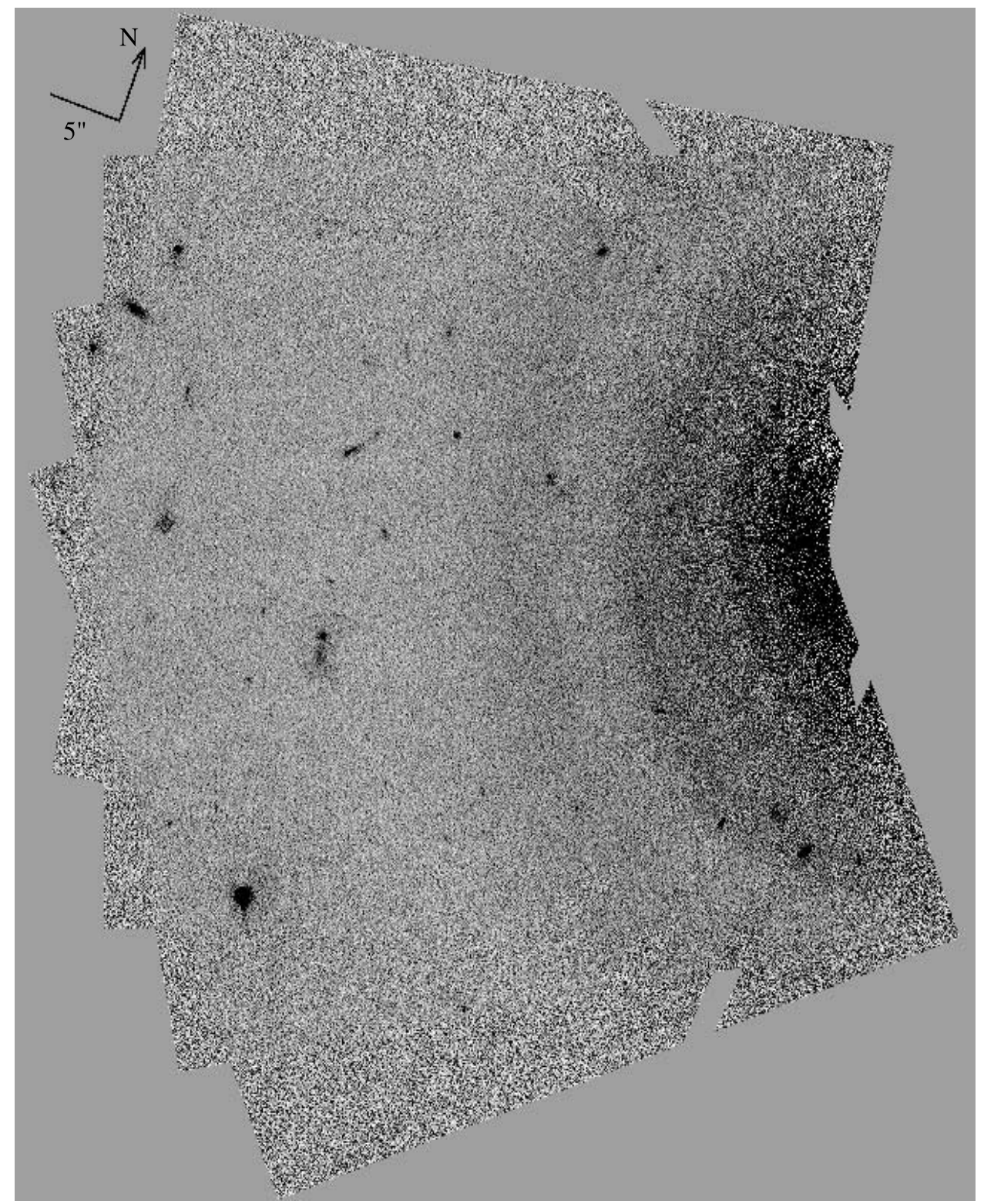

FIG. 2.-Result of subtracting the two stars' PSF assuming a normalization factor of 0.834 . This normalization leaves a central excess as well as two $5^{\prime \prime}$ wide rings with radii of approximately $18^{\prime \prime}$ and $28^{\prime \prime}$.

noise of the star's PSF wings (although the PSF has been subtracted, its Poisson noise still affects the subtracted image). Since surface brightness of the stellar PSF increases steeply closer to the star, our limiting magnitudes become significantly brighter. At distances greater than $30^{\prime \prime}$, the accumulated read noise dominates the total noise. The measured and predicted noise are listed in Table 7. This noise will be somewhat higher in regions that were not observed at all four orientations. Note that a total count rate of $1 e^{-} \mathrm{s}^{-1}$ corresponds to a 50CCD STMAG of 26.405 , and that the magnitude of $\epsilon$ Eri in these same units is 4.02 .

For extremely red objects, as little as $10 \%$ of the total pointsource counts will fall into the central pixel of 50CCD images. For an unambiguous point-source detection, this central pixel should be at least $5 \sigma$ above the rms noise. These assumptions lead to the detectability limits given in the final column of Table 7.

For extended objects, the entire flux should be considered, but a $5 \sigma$ detection will still be required. If noise in different pixels were uncorrelated, then the upper limit for detecting a fixed value of the surface brightness would decrease as the square root of the area. For very large areas, however, any low spatial frequency noise sources would limit the practically achievable faint limit.

We empirically tested the real faint limit for extended sources by masking out discrete sky objects and then comparing the measured background counts in a number of separate regions at various distances from $\epsilon$ Eri in the rollsubtracted image. Taking the standard deviation of the mean fluxes in boxes of a given size at a given distance as the $1 \sigma$ error in the background measurement together with the Poisson noise from the potential source, we derive the $5 \sigma$ detection limits given in Table 8 . For boxes $<0.5 \times 0$ " 5 , we found about the variance expected from scaling the point-to-point fluctuations by $n_{\mathrm{pix}}^{-1 / 2}$, but for larger areas, the fluctuations were bigger than would be expected from Poisson statistics. For example, when averaging over a $3.5 \times 3.5$ box at $20^{\prime \prime}$, the measured pixel-to-pixel variance would imply a $5 \sigma$ detection limit of 


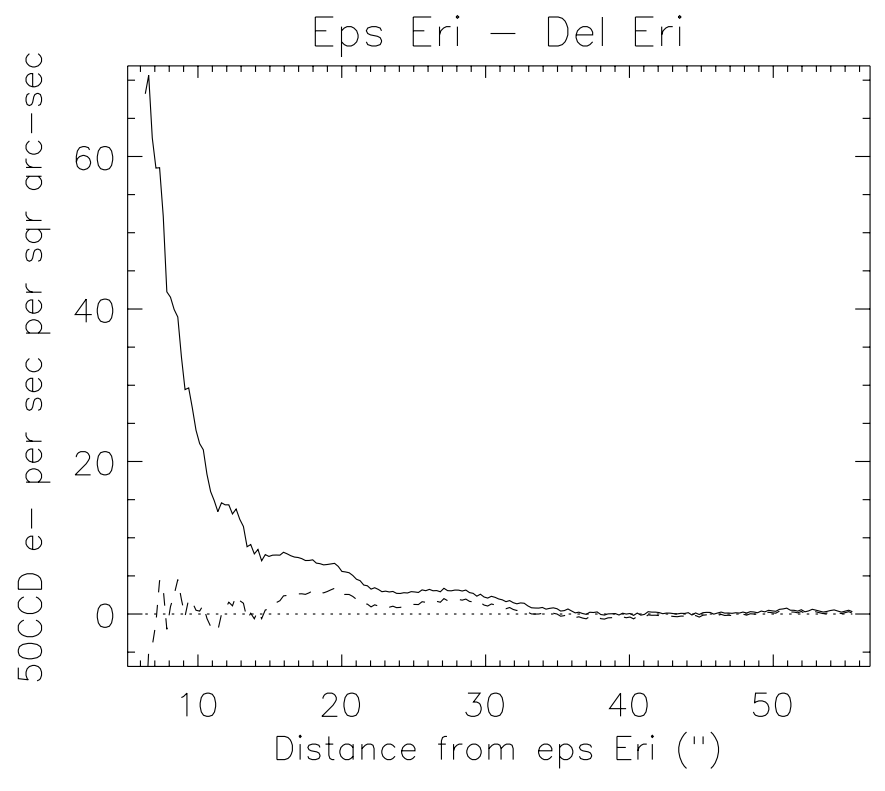

FIG. 3.- Solid line shows the mean count rate $\left(e^{-} \operatorname{arcsec}^{-2} \mathrm{~s}^{-1}\right)$ as a function of radius from $\epsilon$ Eri, after subtracting the $\delta$ Eri PSF with a normalization factor of 0.834 (see Fig. 2). If we change this normalization factor to 0.842 (dashed line), then the overall gradient is minimized, but the rings remain, as shown in Fig. 5.

27.2 STMAG $_{\operatorname{arcsec}}{ }^{-2}$, about 1 mag fainter than the directly measured limit. This difference could be due in part to real sources on the sky, but the lack of a clear correlation of these fluctuations with the submillimeter map or distance from the star requires us to treat such fluctuations as noise.

Note that at $20^{\prime \prime}$ from the star, the shift in position from a $10^{\circ}$ roll change is about $3^{\prime \prime} .5$. Circular or arclike structures much larger than the roll separation will tend to be washed out in the roll-subtracted image. Unfortunately, long, narrow arclike structures oriented in the tangential direction are precisely the kind of structures that are most likely in circumstellar debris disks. This limits the utility of the roll-subtracted image for detecting circumstellar structures much larger than a few arcseconds in extent.

\section{MODELING THE DUST IN THE $\epsilon$ ERI SYSTEM}

\subsection{The Size Distribution of Dust in Circumstellar Debris Disks}

Collisional fragmentation in a sufficiently dense debris disk will lead to a collisional cascade that generates a wide spectrum of particle sizes. Wyatt et al. (1999) discussed extensively the processes that influence the resulting size distribution. Theoretical arguments predict that, when collisions produce similar fragmentation at all size scales, the cascade leads to a distribution of particle sizes $d n(a) \propto a^{n} d a$ (Dohnanyi 1969; Tanaka et al. 1996), with $n \simeq-3.5$. Such a distribution has most of the mass concentrated in the biggest objects, but with most of the surface area being dominated by particles near the lower end of the size distribution. When the emissivity is constant for all sizes, then the observable characteristics will be dominated by the smallest particles.

The distribution may, however, be considerably flattened if the orbital evolution of the smaller particles affects them rapidly enough. The lower cutoff to the distribution is set either by the size at which the timescale for PoyntingRobertson driven orbital decay is smaller than the lifetime of these particles against collisional creation, or by when the

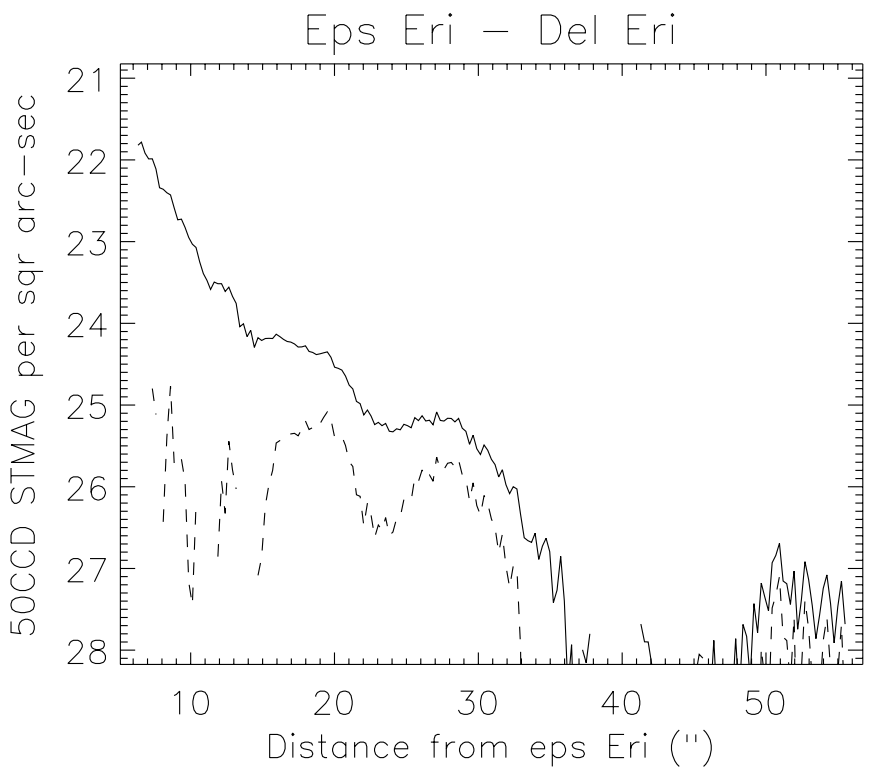

FIG. 4.-Same as Fig. 3, but in units of STMAG $\operatorname{arcsec}^{-2}$.

smallest grains can be blown out of the system by radiation pressure.

A small particle broken off a large object in a circular orbit will be on an unbound hyperbolic orbit when the ratio of radiation to gravitational forces $\beta_{\text {rad }}>0.5$. We can calculate $\beta_{\text {rad }}$ using the same Mie calculations and stellar spectrum used to calculate the dust spectrum (see $\S 5.2 .1$ ).

The time for Poynting-Robertson orbital decay is proportional to the ratio of gravitation to radiative forces on a dust grain. The timescale for Poynting-Robertson drag to change a circular orbit from a radius $r_{1}$ to $r_{2}$ is (Burns et al. 1979)

$$
t_{\mathrm{PR}}=400\left(M_{\odot} / M_{*}\right)\left(r_{1}^{2}-r_{2}^{2}\right) / \beta_{\mathrm{rad}} \mathrm{yr},
$$

where $r_{1}$ and $r_{2}$ are given in AU. For numerical comparisons we define the time needed for the orbit to decay by $20 \%$ of its initial radius as the timescale for Poynting-Robertson drag; this is $36 \%$ of the time to decay to zero radius. At a distance of 60 AU from $\epsilon$ Eri, and assuming the age of the system to be about 0.5-1 Gyr, the Poynting-Robertson effect would be expected to remove any grains smaller than $\approx 100 \mu \mathrm{m}$ unless the disk is dense enough that the small particles are still being generated in collisions.

For the smallest particles in a debris disk, Wyatt et al. (1999) estimated that the collisional timescale is of the order of $t_{\text {orb }} / 4 \pi \tau$, where $t_{\text {orb }}$ is the orbital period and $\tau$ is the effective face-on optical depth (i.e., the geometric filling factor). They also estimated that particles will only be destroyed in collisions with particles larger than a factor of $0.03\left[\left(M_{\odot} / M_{*}\right)\left(r / r_{\oplus}\right)\right]^{1 / 3}$ times their own size (about $12 \%$ at 60 AU from $\epsilon$ Eri), so we define $\tau_{d}$ to be a function of the particle size by integrating only over the appropriate size range; the timescale in years for fragmenting collisions is then:

$$
\begin{aligned}
t_{d} & =\frac{\sqrt{\left(r / r_{\oplus}\right)^{3}\left(M_{\odot} / M_{*}\right)}}{4 \pi \tau_{d}} \\
& \simeq 3.7 \times 10^{5}\left[\left(\frac{r}{60 r_{\oplus}}\right)^{3 / 2}\left(\frac{M_{*}}{M_{\odot}}\right)^{-1 / 2}\left(\frac{\tau_{d}}{10^{-4}}\right)^{-1}\right] \mathrm{yr} .
\end{aligned}
$$




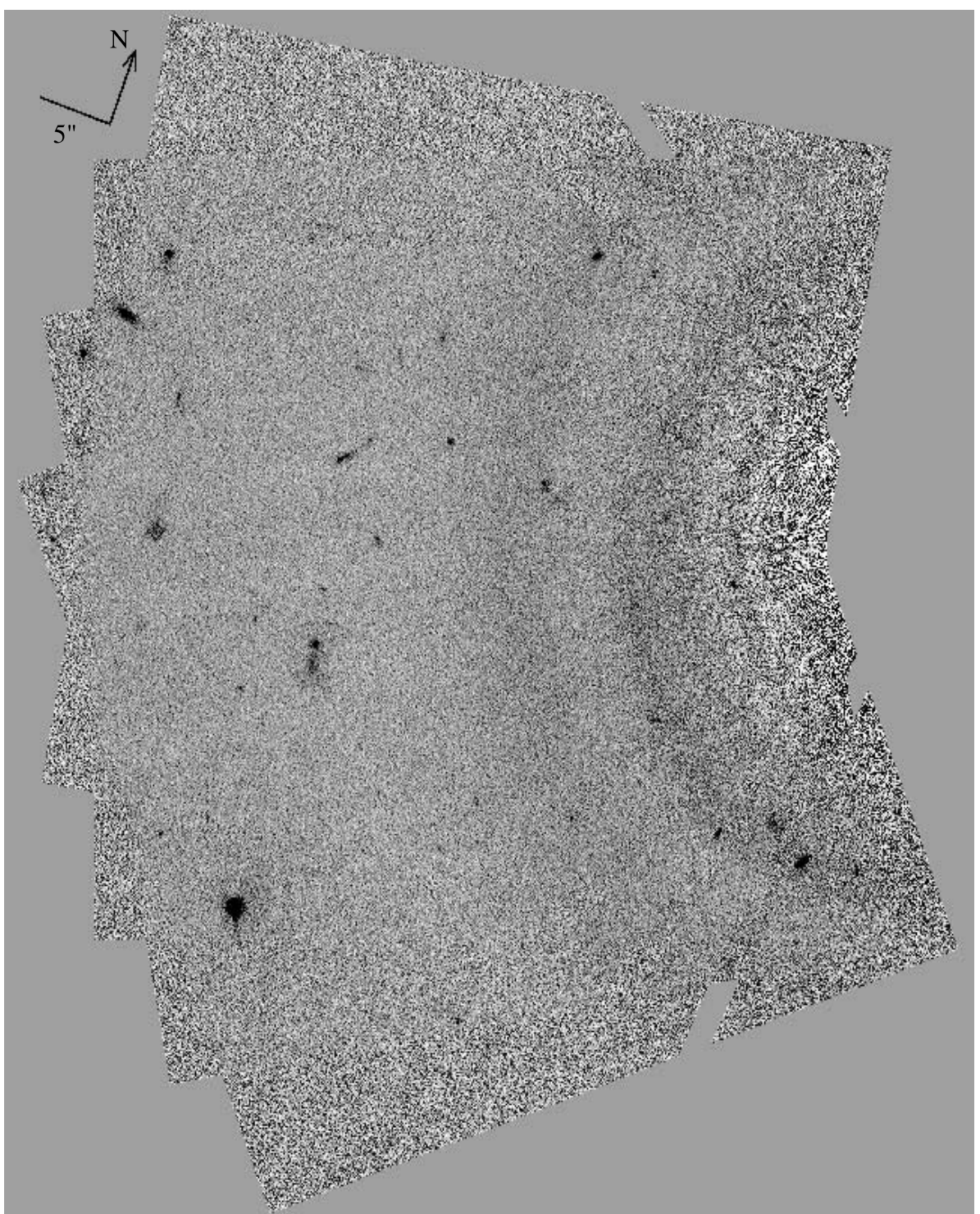

FIG. 5.- Result of subtracting the two stars' PSF assuming a normalization factor of 0.842 . This normalization minimizes the mismatch in flux close to the star, but the two broad rings remain.

For a dust model to be self-consistent, this timescale should be shorter than $t_{\mathrm{PR}}$ for even the smallest particles in the model. We show below that this condition is easily satisfied for the debris disk around $\epsilon$ Eri.

\subsection{Calculation of Dust Models}

\subsubsection{Calculating the Spectrum of Optically Thin Circumstellar Dust}

Given an assumed grain composition and the associated wavelength-dependent optical constants, we performed standard Mie theory calculations for spherical particles covering a wide range of radii $a$ using the code of Wiscombe $(1979,1980)$. This gives, among other quantities, the standard scattering and absorption coefficients $Q_{\lambda, \text { sca }}$ and $Q_{\lambda, \text { abs. }}$. (The emission coefficient at each wavelength $Q_{\lambda \text {, em }}=Q_{\lambda, \text { abs }}$ ).

For the dust calculations, we adopt the flux from a standard solar abundance Kurucz model atmosphere with $T_{\text {eff }}=5180 \mathrm{~K}$ and $\log g=4.75$, normalized to a total stellar luminosity of $0.35 L_{\odot}$. The dust is assumed to be optically thin, and the temperature of each dust grain can be determined by the equilibrium between absorbed stellar radiation and thermal emission. Once the temperature of the grain is determined, then we can calculate the total light from a dust grain at each wavelength as the sum of the scattered starlight and the thermal emission. We assume that the dust grain is small enough that the thermal emission is isotropic. The scattered light, however, will be highly anisotropic. The angular phase function $f(\theta)$ for this scattering can be easily derived from the Mie calculation, and we normalize this function so that $f(\theta)=1$ for isotropic scattering. Then, when viewed at a scattering angle $\theta$, the apparent flux of the dust grain will be

$$
F_{\lambda, g}=\pi a^{2} Q_{\lambda, \text { sca }} F_{\lambda, d} f(\theta)+4 \pi a^{2} \pi B_{\lambda}(T) Q_{\lambda, \text { em }} .
$$

This result will be used below to calculate the expected optical spectrum for a given dust distribution.

\subsubsection{Dust Composition and Porosity}

The observable dust particles in circumstellar debris disks are presumed to be collisionally produced fragments of larger 


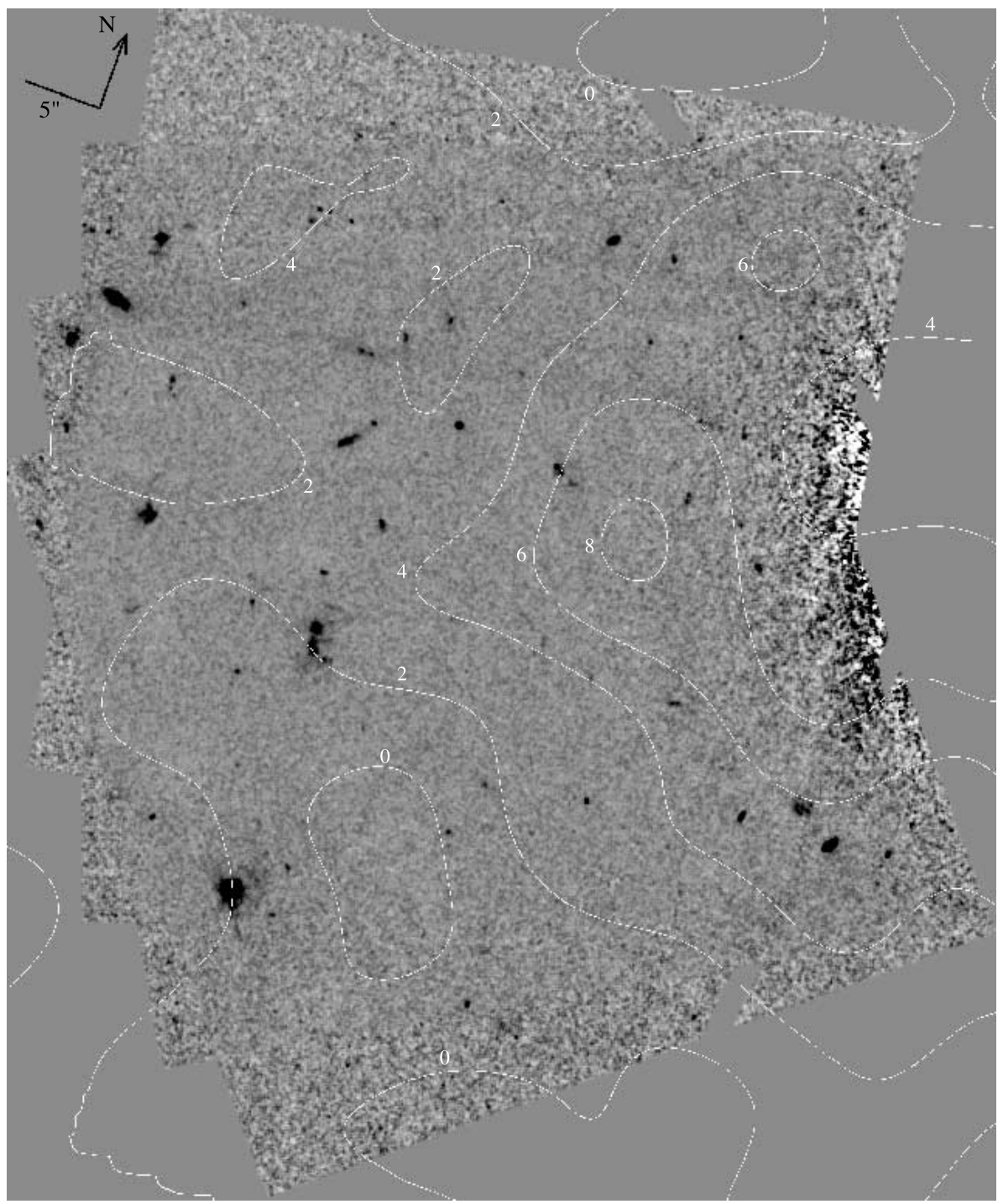

FIG. 6.-A $5 \times 5$ boxcar-smoothed version of the roll-subtracted data compared with the submillimeter contour map, assuming the submillimeter emission shares $\epsilon$ Eri's proper motion. The white numerals give the value of Greaves et al.'s (1998) $850 \mu \mathrm{m}$ contour levels in units of mJy per SCUBA beam area.

objects resembling those in the solar system's Kuiper Belt. These larger objects were probably formed as very porous agglomerations of interstellar grains early in the history of the system.

For the composition and optical properties of these grains, we adopt the model of interstellar and circumstellar dust developed by Li \& Greenberg $(1997,1998)$, which assumes that the dust grains are a mixture of silicates, organic refractories, and voids, with some fraction of the voids possibly filled in by water ice. The Bruggemann mixing rule (Krügel 2003) is used to calculate the effective optical constants for the resulting mixtures from the optical constants of organic refractories and amorphous silicates given by Li \& Greenberg (1997), and that of vacuum.

Wyatt \& Dent (2002) were able to fit the submillimeter and IRAS data for the debris ring around the $\mathrm{A} 3 \mathrm{~V}$ star Fomalhaut by assuming solid (nonporous) grains consisting of $1 / 3$ silicate and $2 / 3$ organic refractory material by volume, and a grain size distribution close to the theoretically expected $n^{-3.5}$ that extends down to the radiation blowout limit for that star. While they could not completely exclude models with some degree of porosity in the grains, they argued that the collisional fragmentation should have resulted in significant compaction of the grains despite the high porosity expected in the primordial parent bodies.

In contrast, Li \& Greenberg $(1997,1998)$ and Li \& Lunine (2003a, 2003b) favor models for the debris disks around HD 141569A, $\beta$ Pictoris, and HR 4796A that assume highly porous grains, with vacuum fractions $P \approx 0.7$ to 0.9 . In some cases, these require a dust size distribution close to $d n \propto$ $a^{-3} d a$, significantly flatter than the theoretically expected $d n \propto$ $a^{-3.5} d a$.

Li et al. (2003) have recently fitted such a model to the available data for $\epsilon$ Eri. They find that a model assuming highly porous particles, and a rather flat size distribution, can provide an excellent fit to both the IRAS and submillimeter data. They assumed that the same dust-size distribution function applies at all distances from the star, and only varied the total number density of grains as a function of radius to match the distribution of the observed $850 \mu \mathrm{m}$ flux. It is not clear whether or not this is realistic. Moro-Martín's \& Malhotra's $(2002,2003)$ dynamical studies of dust produced in our own solar system's Kuiper Belt found that the size distribution function is expected to change substantially as a function of radius. However, the 


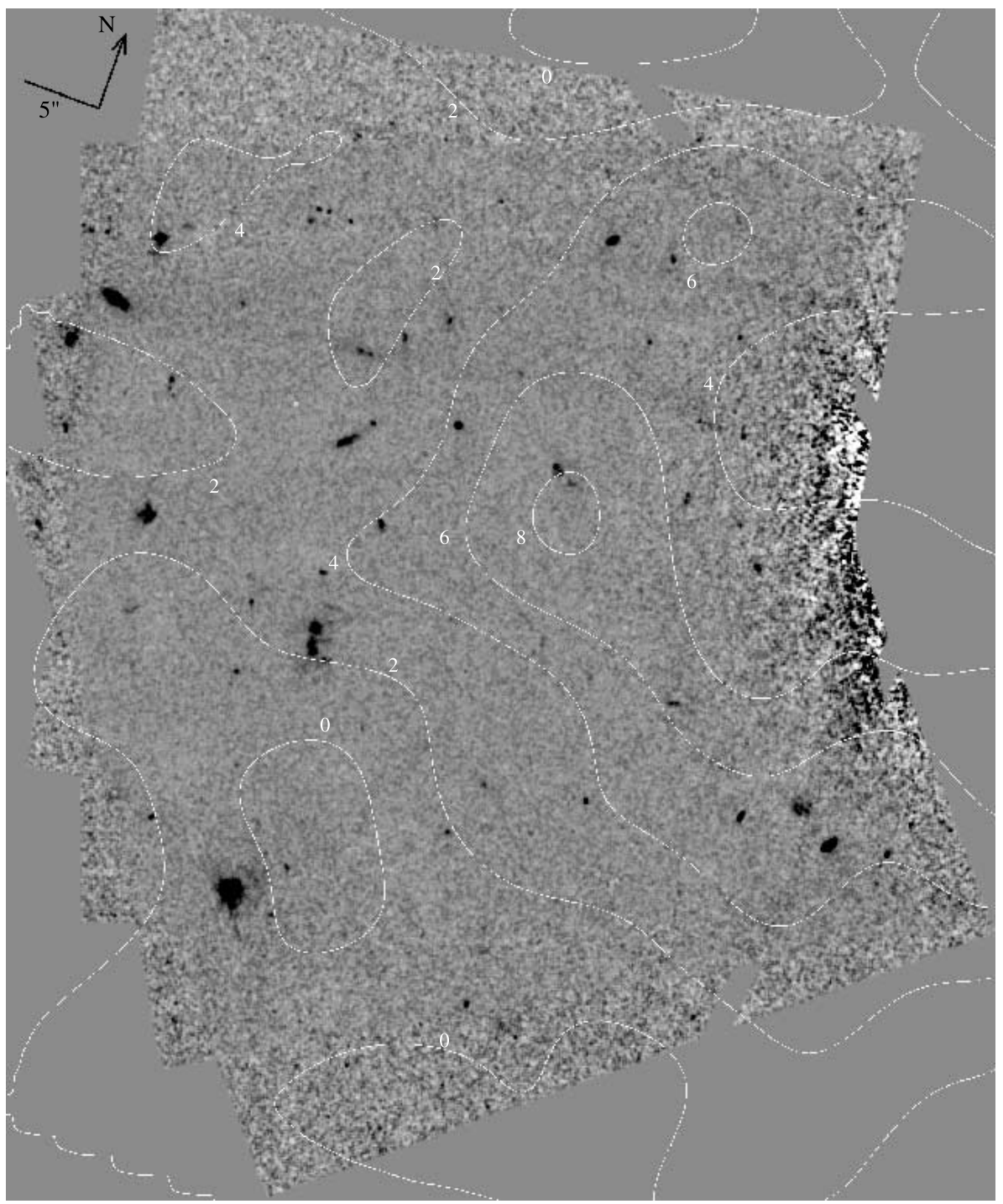

FIG. 7.-Same as Fig. 6, but without correcting the submillimeter contours for the proper motion of $\epsilon$ Eri.

Li et al. (2003) model does give an excellent fit to both the total submillimeter and IRAS fluxes observed in the $\epsilon$ Eri system.

This model assumes $d n \propto a^{-3.1} d a$, lower and upper size limits to the distribution $a_{1}=1 \mu \mathrm{m}$ and $a_{2}=1 \mathrm{~cm}$, and a porosity $P=0.9$. The solid portion of the grains is assumed to consist of an organics/silicate mix in a 58:42 ratio by volume. The radial distribution of dust density is modeled as a Gaussian centered at $55 \mathrm{AU}$, with a FWHM of $30 \mathrm{AU}$. We repeated Li et al's calculations for the flux from such a dust distribution, but also included the contribution of the scattered light, assuming a scattering angle of $90^{\circ}$ (i.e., a face-on disk, as indicated by the morphology of the $850 \mu \mathrm{m}$ flux).

It is instructive to examine the radiative forces and timescales for this model of the dust distribution. Figure 9 shows $\beta_{\text {rad }}$, the ratio of radiative to gravitational forces, for dust grains of the modeled composition in the $\epsilon$ Eri system (see also Fig. 3 of Sheret et al. 2004). At large grain sizes, the low density of porous grains substantially increases the effects of radiation pressure relative to that on solid grains of the same composition and size. At small sizes, however, the porous grains no longer effectively scatter radiation, and $\beta_{\text {rad }}$ drops below that of solid grains, never becoming large enough $\left(\beta_{\text {rad }}>0.5\right)$ for such grains to be efficiently blown out of the system. In reality, it is likely that the grains become less porous as they are fragmented to very small sizes. In any case, the timescale for fragmenting collisions expected for the model of $\mathrm{Li}$ et al. (2003) is much shorter than $t_{\mathrm{PR}}$ at all sizes (Fig. 10), so small grains should be abundant, but predicting the detailed distribution of grain size and porosities at the lower end of the distribution will be difficult.

Attempts made to fit solid-grain models $(P=0)$ have not resulted in as good a fit as the $\mathrm{Li}$ et al. (2003) model. However, Sheret et al. (2004) found that a simple model of a thin ring at $60 \mathrm{AU}$ with solid silicate/organic grains, $a_{1}=1.75 \mu \mathrm{m}$, $a_{2}=5 \mathrm{~m}$, and $d n \propto a^{-3.5} d a$, fits the IR and submillimeter data with a reduced $\chi^{2}$ of 2.8 .

\subsubsection{Optical Brightness of Dust Models Normalized to Mean Submillimeter Flux}

Near 55 AU from $\epsilon$ Eri, the typical $850 \mu \mathrm{m}$ surface brightness observed by Greaves et al. (1998) is $\sim 0.02 \mathrm{mJy} \mathrm{arcsec}^{-2}$. After subtracting the mean emission of the ring, the $850 \mu \mathrm{m}$ flux in the brightest clump totals to $\sim 2.6 \mathrm{mJy}$. If the angular 


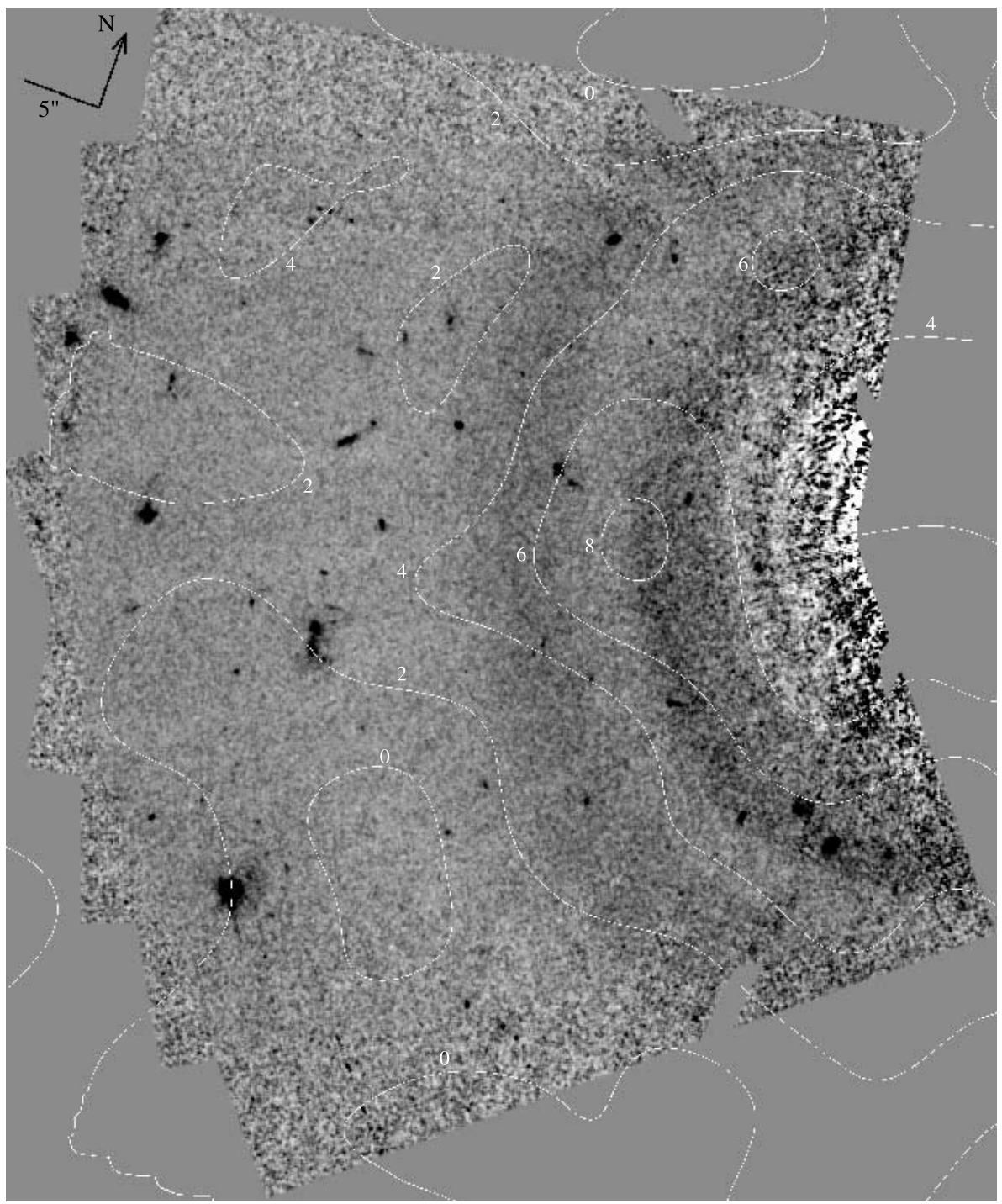

FIG. 8. - Same as Fig. 6, except that here the contours are compared with the sky image made by subtracting the PSF derived from the $\delta$ Eri observations (see Fig 5).

extent of the clump is comparable to the SCUBA beam size, this amounts to a surface brightness enhancement of an a additional $0.015 \mathrm{mJy} \operatorname{arcsec}^{-2}$.

In Figure 11, we show, for a dust model with the parameters of $\mathrm{Li}$ et al. (2003), the calculated total SED for the whole dust cloud, and also for just the dust at 55 AU. Normalizing this model to an $850 \mu \mathrm{m}$ surface brightness at $55 \mathrm{AU}$ of
$0.02 \mathrm{mJy} \operatorname{arcsec}^{-2}$ yields a predicted STIS 50CCD surface brightness of 27.9 STMAG $\operatorname{arcsec}^{-2}$ (equivalent to $6.5 \times$ $10^{-4}$ counts pixel ${ }^{-1} \mathrm{~s}^{-1}$ ).

If we instead assume the parameters of the Sheret et al. (2004) solid-grain model, we predict an optical 50CCD surface brightness of 24.5 STMAG - rather brighter than the rings in the directly subtracted image.

TABLE 7

50CCD Point Source Detection Limits in Roll-subtracted Image

\begin{tabular}{|c|c|c|c|}
\hline $\begin{array}{l}\text { Distance } \\
(\operatorname{arcsec})\end{array}$ & $\begin{array}{c}1 \sigma \text { Measured Noise } \\
\left(e^{-} \text {pixel }^{-1} \mathrm{~s}^{-1}\right)\end{array}$ & $\begin{array}{c}1 \sigma \text { Predicted Noise } \\
\left(e^{-} \text {pixel }^{-1} \mathrm{~s}^{-1}\right)\end{array}$ & $\begin{array}{c}5 \sigma \text { Point Source } \\
\text { Limiting Magnitude }\end{array}$ \\
\hline 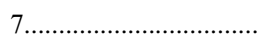 & 0.064 & 0.052 & 25.0 \\
\hline 8 & 0.055 & 0.043 & 25.3 \\
\hline 9........................................ & 0.041 & 0.037 & 25.5 \\
\hline $10 \ldots \ldots \ldots \ldots$ & 0.033 & 0.032 & 25.7 \\
\hline $12 \ldots \ldots \ldots \ldots$ & 0.023 & 0.026 & 26.1 \\
\hline 15 & 0.017 & 0.021 & 26.3 \\
\hline $20 \ldots \ldots$ & 0.013 & 0.017 & 26.6 \\
\hline $30+\ldots$ & 0.012 & 0.015 & 26.7 \\
\hline
\end{tabular}


TABLE 8

50CCD Extended Source $5 \sigma$ Surface Brightness Limits in Roll-subtracted Image

\begin{tabular}{|c|c|c|c|c|}
\hline \multirow{2}{*}{$\begin{array}{c}\text { DISTANCE } \\
\text { FROM STAR } \\
(\operatorname{arcsec})\end{array}$} & \multicolumn{4}{|c|}{$5 \sigma$ Limiting Surface Brightness (STMAG $\operatorname{arcsec}^{-2}$ ) vs. Box Siz } \\
\hline & $0.5 \times 0.5$ & $1^{\prime \prime} \times 1^{\prime \prime}$ & $3.5 \times 3.5$ & $5^{\prime \prime} \times 5^{\prime \prime}$ \\
\hline $15 \ldots$ & 24.85 & 25.34 & 26.61 & 27.29 \\
\hline $20 \ldots \ldots \ldots \ldots \ldots \ldots \ldots \ldots$ & 25.06 & 25.60 & 26.20 & 26.92 \\
\hline 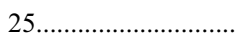 & 25.39 & 26.00 & 27.01 & 27.33 \\
\hline 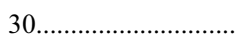 & 25.46 & 26.28 & 27.15 & 27.25 \\
\hline 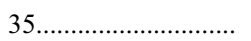 & 25.33 & 26.04 & 26.90 & 26.97 \\
\hline
\end{tabular}

In the roll-subtracted image, any tangential feature larger than the roll change between images will show up in the PSF rather than in the sky image, and so circular rings would be invisible. The bright clump might be detectable if it were not too diffuse or too spread out in the tangential direction. If the dust parameters in the clump are the same as in the rest of the ring, we would predict a total 50CCD optical brightness of 22.6 STMAG. Spread over a SCUBA beam area, this would give a surface brightness of 28 STMAG $\operatorname{arcsec}^{-2}$, well below our most optimistic detection limits of $\approx 27$ STMAG $\operatorname{arcsec}^{-2}$. The clump would have to be concentrated within an area of no more than $1 / 4$ of the SCUBA beam size before we would expect to see it.

This assumes that the dust distribution in the clump is the same as that of the ring as a whole. If the clump was created by resonant interactions of dust with a Neptune-like planet in the $\epsilon$ Eri system, smaller particles, which are strongly perturbed by radiation pressure, may be less likely to collect in the same resonances. If, for the enhancement in the clump, we change the lower limit of the size distribution to be $150 \mu \mathrm{m}$, and again normalize to $0.015 \mathrm{mJy} \operatorname{arcsec}^{-2}$ at $850 \mu \mathrm{m}$, then the predicted surface brightness of the clump drops to $\sim 29.8$ STMAG $\operatorname{arcsec}^{-2}\left(1.5 \times 10^{-4}\right.$ counts pixel $\left.{ }^{-1} \mathrm{~s}^{-1}\right)$. Such a model also substantially reduces the clump's contrast against the rest of the ring in the thermal IR.

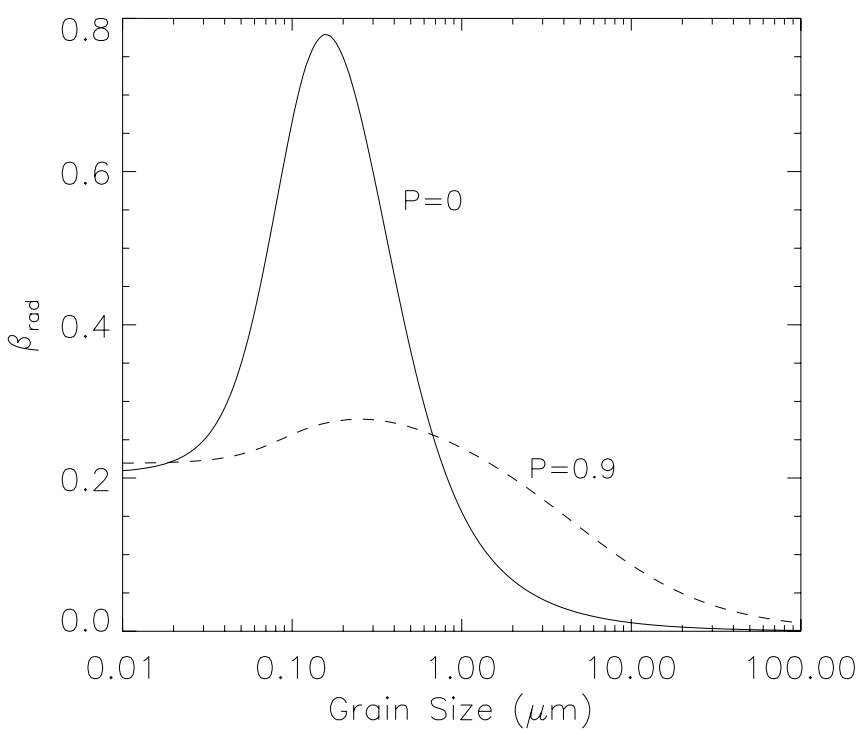

FIG. 9.-Ratio of radiative to gravitational forces, $\beta_{\text {rad, }}$, in the $\epsilon$ Eri system for the porous grains assumed by Li et al. (2003), plotted as a function of grain size (dashed line). For comparison, $\beta_{\text {rad }}$ for solid grains of the same composition is also plotted (solid line).
The rings seen in the directly subtracted image ( $\epsilon$ Eri$\delta$ Eri), with a surface brightness of about 25 STMAG $\operatorname{arcsec}^{-2}$, are much brighter than the predictions of Li et al. (2003), but slightly fainter than predicted by the model of Sheret et al. (2004). If the rings are PSF subtraction artifacts, they are bright enough to obscure the expected signal from the dust. If real, they would imply a much larger abundance of small grains that scatter efficiently in the optical than does the model of $\mathrm{Li}$ et al. The lack of obvious counterparts in the optical ring corresponding to the submillimeter clumpiness might be explained by the very different dynamical behavior of the small grains.

Unfortunately, available modeling of the HST STIS PSF at distances of $20^{\prime \prime}$ is inadequate to provide a clear answer regarding the reality of the ringlike features seen after the subtraction of the two stars' PSFs.

\subsubsection{Variations of Models}

The models of both Li et al. (2003) and Sheret et al. (2004) assume that all excess IR and submillimeter flux in the $\epsilon$ Eri system is due to a single dust distribution that is well traced by the $850 \mu \mathrm{m}$ emission. If a substantial fraction of the IR excess is instead due to an inner zodiacal cloud of particles that contributes little at $850 \mu \mathrm{m}$, then constraints on possible

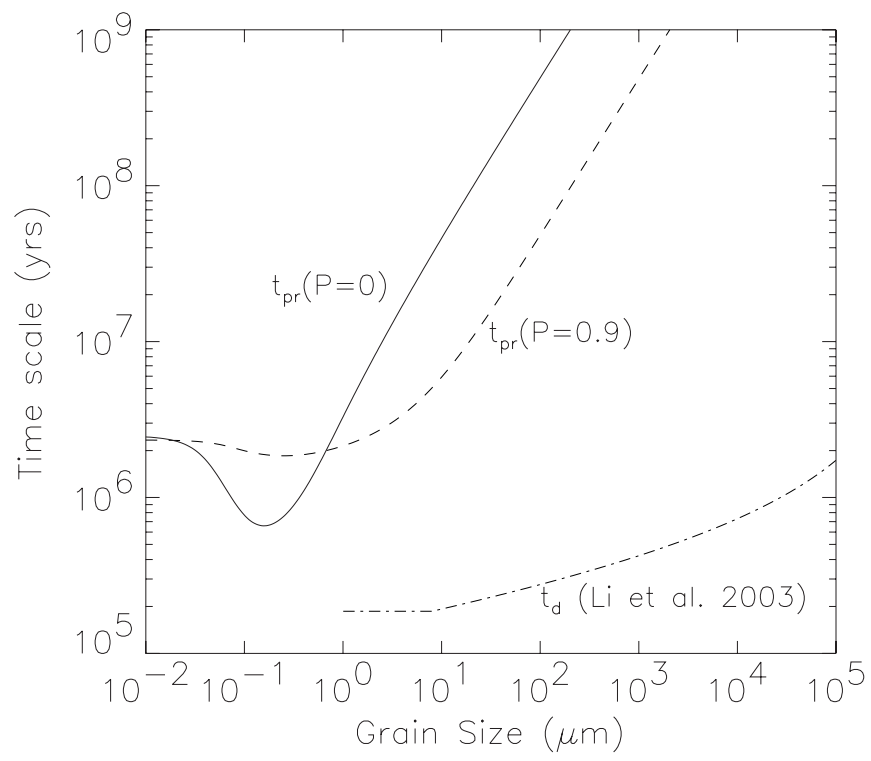

FIG. 10.-Poynting-Robertson orbital decay timescale compared for solid $(P=0)$ and porous $(P=0.9)$ grains. This timescale is always much larger than the timescale for the fragmentation rate of grains $\left(t_{d}\right)$ we calculate for the parameters of the Li et al. (2003) dust model. 


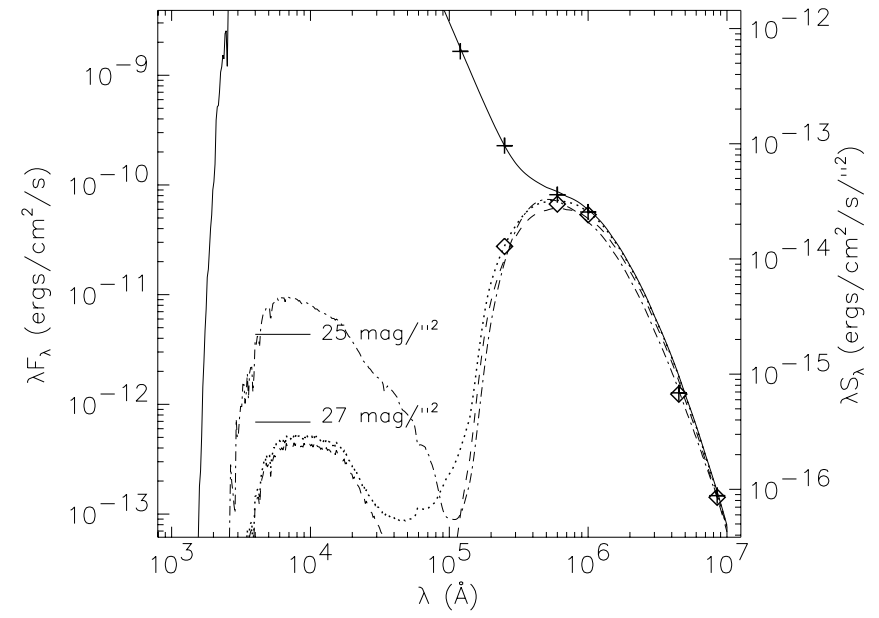

FIG. 11.-Dotted line: Our calculation of the total spectral energy distribution for Li et al's (2003) favored model for the dust ring around $\epsilon$ Eri, including the contribution from scattered optical light. Solid line: This model added to the stellar SED. Crosses: Observed IRAS and submillimeter flux observations. Diamonds: Flux values after subtracting the star light. Dashed line: SED of the surface brightness of this model at 55 AU (surface brightness values given on right axis), with the scales shifted to overlap the curves at $850 \mu \mathrm{m}$. Dash-dotted line: SED for the parameters of the thin-ring solid-grain model of Sheret et al. (2004). Also marked are the approximate surface brightness levels corresponding to STIS 50CCD brightnesses of 25 and $27 \mathrm{mag} \operatorname{arcsec}^{-2}$. These levels roughly correspond to the brightness of the rings seen after the direct PSF subtraction, and the upper limit to the surface brightness determined for the roll-subtracted PSF image, respectively.

models are considerably relaxed, although the IRAS measurements will still provide an upper limit to the allowed IR flux from the 55 AU ring.

For example, if we change the model of Li et al. (2003) by simply allowing the upper limit of the size distribution to extend to $10^{7} \mu \mathrm{m}$ instead of $10^{4} \mu \mathrm{m}$, and adjust the overall normalization to again match the observed $850 \mu \mathrm{m}$ flux, then both the predicted IRAS band and optical fluxes drop by a factor of about 4 . If we instead assume Li et al's parameters, but with solid rather than porous grains, the predicted IRAS fluxes drop by a factor of $2-3$, but the optical surface brightness increases by a factor of 4 . However, without better information on the spatial distribution of the $10-100 \mu \mathrm{m}$ flux it is difficult to choose among the different possible models. Such information will eventually be provided by Spitzer Space Telescope images of the $\epsilon$ Eri system. However, there is still unique information about the distribution of small grains that would be provided by direct detection of the optical scattered light that cannot be obtained from even the most detailed observations of the thermal dust emission.

The simple models discussed here clearly have some limitations. Real dust does not consist of the perfectly smooth spheres assumed in Mie theory, but will have considerable surface roughness. For example, Lisse et al. (1998) in a study of cometary dust found that considering the expected fractal structure of the dust could increase the optical scattering at $90^{\circ}$ by as much as a factor of 3 . In addition, the porosity is unlikely to be the same for all grain sizes. Even if the parent bodies are highly porous, at sufficiently small sizes the dust may either be significantly compacted by collisions, or will have broken up into smaller but more solid component particles. However, currently there are insufficient observational data for the $\epsilon$ Eri dust ring to constrain the additional free parameters needed by such models. This situation will improve substantially when Spitzer images of this system become available.

\section{CONCLUSIONS}

Our deep optical observations of the $\epsilon$ Eri submillimeter ring have not provided clear evidence for detection of an optical counterpart. The upper limits measured are consistent with existing models of the dust in the submillimeter ring, and provide some constraints on the nature and amount of the smallest dust grains. Our optical limits should provide tighter constraints once Spitzer images are available for this system.

We found approximately 59 objects between 12 ".5 and $58^{\prime \prime}$ from $\epsilon$ Eri, with brightnesses between 19.8 and 28 mag (STIS 50CCD STMAG). If any of the more compact of these objects were associated with the $\epsilon$ Eri system, they would correspond to brown dwarfs of $\approx 0.03-0.05 M_{\odot}$. However, it is much more likely that the majority of these objects are background stars and galaxies unrelated to the $\epsilon$ Eri system. A secondepoch HST observation of comparable depth would immediately determine whether any of these objects shares $\epsilon$ Eri's 0 ".98 $\mathrm{yr}^{-1}$ proper motion.

Support for proposal GO-09037 was provided by NASA through grants from the Space Telescope Science Institute, which is operated by the Association of Universities for Research in Astronomy, Inc., under NASA contract NAS $5-26555$.

\section{APPENDIX}

Table 9 contains a list of detected objects. Some of the listed objects close to the star may well be noise or PSF-subtraction artifacts, while other faint but real objects may be omitted from this list. For each object the J2000 coordinates at epoch 2002.071 , the distance from $\epsilon$ Eri, the approximate size of the object, and the total brightness in STIS 50CCD STMAG units are listed. For compact objects, the size given is the FWHM from a Moffat fit, while for more extended objects the dimensions given are an approximate estimate. One STIS CCD pixel corresponds to about 0 ".05071 on the sky.

Macintosh et al. (2003), performed a $K$-band adaptive optics search for close companions around $\epsilon$ Eri, and found 10 candidates, although none are proper-motion companions to $\epsilon$ Eri. Four of these objects lie in our field of view. Objects 4 and 6 correspond to extended galaxies and are noted in the table. Their objects 5 and 9 have no optical counterparts. 
TABLE 9

Detected Objects near $\epsilon$ Eri, Ordered by Distance from the Central Star

\begin{tabular}{|c|c|c|c|c|c|}
\hline $\begin{array}{c}\text { R.A. } \\
\text { (J2000; Epoch } \\
\text { 2002.07) }\end{array}$ & $\begin{array}{c}\text { Decl. } \\
\text { (J2000; Epoch } \\
\text { 2002.07) }\end{array}$ & $\begin{array}{c}\text { Distance } \\
(\operatorname{arcsec})\end{array}$ & $\begin{array}{c}\text { Size } \\
\text { (pixels) }\end{array}$ & $\begin{array}{c}\text { 50CCD } \\
\text { STMAG }\end{array}$ & Notes \\
\hline $33256.4185 \ldots \ldots \ldots \ldots$ & -92736.314 & 12.563 & $3 \times 6$ & 25.2 & \\
\hline $33256.5551 \ldots \ldots \ldots \ldots$ & -92734.156 & 13.517 & 2.3 & 26.7 & \\
\hline $33256.4763 \ldots \ldots \ldots$. & -92721.908 & 14.150 & 3.8 & 25.4 & \\
\hline $33256.6711 \ldots \ldots \ldots .$. & -92728.703 & 14.602 & 7 & 25.9 & \\
\hline $33256.8063 \ldots \ldots \ldots \ldots$ & -92733.636 & 16.962 & 12 & 25.7 & double object \\
\hline $33256.8713 \ldots \ldots \ldots$. & -92728.768 & 17.549 & 2.0 & 26.7 & \\
\hline $33256.8330 \ldots \ldots \ldots \ldots$ & -92722.815 & 18.370 & 2.3 & 26.1 & \\
\hline $33255.9059 \ldots \ldots \ldots . .$. & -92749.906 & 20.223 & 26 & 24.2 & fuzzy patch 1.3 diameter \\
\hline $33256.5430 \ldots \ldots \ldots \ldots$ & -92746.244 & 20.642 & $3 \times 7$ & 25.9 & faint line \\
\hline $33255.4660 \ldots \ldots \ldots \ldots$ & -92750.758 & 21.065 & 3.4 & 25.1 & \\
\hline $33255.7102 \ldots \ldots \ldots \ldots$ & -92751.351 & 21.406 & $8 \times 16$ & 23.0 & oval \\
\hline $33256.1177 \ldots \ldots \ldots . .$. & -92751.611 & 22.582 & $5 \times 9$ & 24.4 & oval \\
\hline $33257.1903 \ldots \ldots \ldots .$. & -92725.101 & 22.740 & 2.9 & 26.4 & \\
\hline $33256.5049 \ldots \ldots \ldots$. & -92709.433 & 23.767 & 3.0 & 25.6 & \\
\hline $33257.2179 \ldots \ldots \ldots$ & -92719.614 & 24.862 & 3.2 & 25.8 & \\
\hline $33257.3883 \ldots \ldots \ldots \ldots$ & -92734.610 & 25.586 & 3.0 & 25.1 & extended flux \\
\hline $33257.1796 \ldots \ldots \ldots \ldots$ & -92745.611 & 27.068 & $3 \times 10$ & 26.9 & faint line \\
\hline $33257.4928 \ldots \ldots \ldots . .$. & -92719.772 & 28.554 & $4 \times 6$ & 23.8 & oval galaxy ${ }^{\mathrm{a}}$ \\
\hline $33256.7705 \ldots \ldots \ldots \ldots$ & -92754.110 & 28.991 & 2.3 & 25.4 & \\
\hline $33257.6676 \ldots \ldots \ldots \ldots$ & -92729.800 & 29.281 & 3.4 & 26.2 & \\
\hline $33257.2929 \ldots \ldots \ldots \ldots$ & -92711.811 & 29.844 & 3 & 26.4 & on edge of FOV \\
\hline $33257.8475 \ldots \ldots \ldots \ldots$ & -92734.308 & 32.240 & 4.4 & 24.5 & \\
\hline $33257.2044 \ldots \ldots \ldots \ldots$ & -92755.359 & 33.897 & 3.7 & 26.2 & \\
\hline $33258.0335 \ldots \ldots \ldots \ldots$ & -92728.253 & 34.728 & 6.1 & 26.1 & extended structure mag 24.8 \\
\hline $33258.0057 \ldots \ldots \ldots \ldots$ & -92719.861 & 35.718 & 3.1 & 26.6 & \\
\hline $33258.0150 \ldots \ldots \ldots \ldots$ & -92741.904 & 36.439 & 7.6 & 25.2 & \\
\hline $33258.1936 \ldots \ldots \ldots \ldots$ & -92730.231 & 37.056 & $5 \times 9$ & 25.6 & extended structure \\
\hline $33257.2856 \ldots \ldots \ldots \ldots$ & -92758.997 & 37.445 & 4 & 26.1 & \\
\hline $33258.1967 \ldots \ldots \ldots \ldots$ & -92736.068 & 37.606 & 4 & 26.1 & \\
\hline $33258.2965 \ldots \ldots \ldots \ldots$ & -92737.911 & 39.394 & $4 \times 10$ & 24.1 & long oval \\
\hline $33258.3564 \ldots \ldots \ldots \ldots$ & -92731.950 & 39.514 & 1.5 & 25.6 & extended structure \\
\hline $33258.1802 \ldots \ldots \ldots . .$. & -92746.132 & 40.259 & 4.0 & 26.1 & \\
\hline $33258.3193 \ldots \ldots \ldots \ldots$ & -92717.338 & 40.889 & 4 & 27.8 & \\
\hline $33258.0451 \ldots \ldots \ldots \ldots$ & -92751.423 & 40.946 & 5 & 25.5 & extended structure \\
\hline $33258.1279 \ldots \ldots \ldots \ldots$ & -92749.574 & 41.079 & 3.2 & 23.2 & double irr galaxy ${ }^{\mathrm{b}}$ \\
\hline $33258.5861 \ldots \ldots \ldots \ldots$ & -92724.382 & 43.210 & 2.0 & 27.4 & \\
\hline $33256.9571 \ldots \ldots \ldots .$. & -92808.992 & 43.308 & 3.7 & 25.3 & \\
\hline $33258.6919 \ldots \ldots \ldots \ldots$ & -92724.300 & 44.772 & 3.2 & 27.1 & extended structure mag 25.8 \\
\hline $33258.4244 \ldots \ldots \ldots \ldots$ & -92749.437 & 44.919 & 3.8 & 25.7 & \\
\hline $33258.7317 \ldots \ldots \ldots .$. & -92724.345 & 45.350 & 2.5 & 26.2 & \\
\hline $33258.7489 \ldots \ldots \ldots$. & -92725.229 & 45.504 & 5 & 26.1 & \\
\hline $33258.6853 \ldots \ldots \ldots . .$. & -92719.098 & 45.620 & 11 & 25.9 & noise? \\
\hline $33258.3840 \ldots \ldots \ldots \ldots$ & -92753.969 & 46.549 & 3 & 25.7 & \\
\hline $33257.8873 \ldots \ldots \ldots \ldots$ & -92804.667 & 47.568 & 2.4 & 26.0 & \\
\hline $33258.9032 \ldots \ldots \ldots \ldots$ & -92731.625 & 47.574 & 2 & 26.1 & \\
\hline $33257.8779 \ldots \ldots \ldots$. & -92807.866 & 49.856 & 5 & 26.0 & \\
\hline $33259.0790 \ldots \ldots \ldots \ldots$ & -92737.894 & 50.771 & 5.2 & 25.4 & \\
\hline $33259.0779 \ldots \ldots \ldots$. & -92738.375 & 50.833 & 3 & 27.1 & \\
\hline $33258.9754 \ldots \ldots \ldots \ldots$ & -92746.790 & 51.449 & 20 & 23.7 & fuzzy patch $\approx 1^{\prime \prime}$ diameter \\
\hline $33258.0864 \ldots \ldots \ldots \ldots$ & -92807.301 & 51.501 & 1.8 & 19.8 & bright star \\
\hline $33258.8881 \ldots \ldots \ldots \ldots$ & -92752.372 & 52.366 & 3.3 & 26.6 & \\
\hline $33259.3340 \ldots \ldots \ldots .$. & -92729.536 & 53.912 & 1.8 & 22.2 & star \\
\hline $33258.5123 \ldots \ldots \ldots \ldots$ & -92804.604 & 54.265 & 3.3 & 25.7 & \\
\hline $33259.4291 \ldots \ldots \ldots \ldots$ & -92734.188 & 55.481 & $10 \times 30$ & 22.6 & oval \\
\hline $33259.3587 \ldots \ldots \ldots \ldots$ & -92716.223 & 55.970 & 4 & 28.3 & \\
\hline $33259.4461 \ldots \ldots \ldots \ldots$ & -92742.934 & 57.069 & $5 \times 10$ & 25.6 & \\
\hline $33259.5610 \ldots \ldots \ldots \ldots$ & -92730.327 & 57.267 & 4 & 26.0 & \\
\hline $33259.5556 \ldots \ldots \ldots . .$. & -92737.599 & 57.698 & 3.5 & 23.5 & oval outer isophote \\
\hline $33259.4106 \ldots \ldots \ldots \ldots$ & -9 2749.428 & 58.392 & 4.2 & 25.1 & \\
\hline
\end{tabular}

${ }^{\text {a }}$ Object 4 from Macintosh et al. (2003), $K=19.4$.

b Object 6 from Macintosh et al. (2003), $K=20.2$. 


\section{REFERENCES}

Aumann, H. H. 1985, PASP, 97, 885

Aumann, H. H., et al. 1984, ApJ, 278, L23

Beichman, C. A., Neugebauer, G., Habing, H. J., Clegg, P. E., \& Chester, T. J. 1985, Infrared Astronomical Satellite (IRAS) Catalogs and Atlases: Explanatory Supplement (NASA STI/Recon Tech. Rep. 85-18898; Pasadena: JPL)

Burns, J. A., Lamy, P. L., \& Soter, S. 1979, Icarus, 40, 1

Chabrier, G., Baraffe, I., Allard, F., \& Hauschildt, P. 2000, ApJ, 542, 464

Chini, R., Krügel, E., \& Kreysa, E. 1990, A\&A, 227, L5

Chini, R., Krügel, E., Kreysa, E., Shustov, B., \& Tutukov, A. 1991, A\&A, 252,220

Dohnanyi, J. S. 1969, J. Geophys. Res., 74, 2431

Gillett, F. C. 1986, in Light on Dark Matter: Proc. First IRAS Conf., ed. F. P. Israel (Dordrecht: Reidel), 61

Gillett, F. C., \& Aumann, H. H. G. 1983, BAAS, 15, 799

Grady, C. A., et al. 2003, PASP, 115, 1036

Greaves, J. S., et al. 1998, ApJ, 506, L133

Hatzes, A. P., et al. 2000, ApJ, 544, L145

Holland, W. S., et al. 1998, Nature, 392, 788

Kim Quijano, J., et al. 2003, STIS Instrument Handbook, Version 7.0 (Baltimore: STScI), http://www.stsci.edu/hst/stis/documents/handbooks/ currentIHB/cover.html

Krist, J. 1993, in ASP Conf. Ser. 52, Astronomical Data Analysis Software and Systems II (San Francisco: ASP), 536 1995, in ASP Conf. Ser. 77, Astronomical Data Analysis Software and Systems IV (San Francisco: ASP), 349

Krügel, E. 2003, The Physics of Interstellar Dust (Bristol: Inst. Physics)

Li, A., \& Greenberg, J. M. 1997, A\&A, 323, 566 1998, A\&A, 331, 291

Li, A., \& Lunine, J. I. 2003a, ApJ, 590, 368
Li, A., \& Lunine, J. I. 2003b, ApJ, 594, 987

Li, A., Lunine, J. I., \& Bendo, G. J. 2003, ApJ, 598, L51

Liou, J., \& Zook, H. A. 1999, AJ, 118, 580

Lisse, C. M., A’Hearn, M. F., Hauser, M. G., Kelsall, T., Lien, D. J., Moseley, S. H., Reach, W. T., \& Silverberg, R. F. 1998, ApJ, 496, 971

Macintosh, B. A., Becklin, E. E., Kaisler, D., Konopacky, Q., \& Zuckerman, B. 2003, ApJ, 594, 538

Mermilliod, J.-C., Mermilliod, M., \& Hauck, B. 1997, A\&AS, 124, 349

Moro-Martín, A., \& Malhotra, R. 2002, AJ, 124, 2305 2003, AJ, 125, 2255

Ozernoy, L. M., Gorkavyi, N. N., Mather, J. C., \& Taidakova, T. A. 2000, ApJ, 537, L147

Pavlovsky, C., et al. 2003, ACS Instrument Handbook, Version 4.0 (Baltimore: STScI), http://www.stsci.edu/hst/acs/documents/handbooks/cycle13/cover.html Schütz, O., Nielbock, M., Wolf, S., Henning, T., \& Els, S. 2004, A\&A, 414, L9 Sheret, I., Dent, W. R. F., \& Wyatt, M. C. 2004, MNRAS, 348, 1282

Soderblom, D. R., \& Dappen, W. 1989, ApJ, 342, 945

Song, I., Caillault, J.-P., Barrado y Navascués, D., Stauffer, J. R., \& Randich, S. 2000, ApJ, 533, L41

Tanaka, H., Inaba, S., \& Nakazawa, K. 1996, Icarus, 123, 450

Quillen, A. C., \& Thorndike, S. 2002, ApJ, 578, L149

Weintraub, D. A., \& Stern, S. A. 1994, AJ, 108, 701

Wiscombe, W. J. 1979, MIE Scattering Calculations, Advances in Technique and Fast, Vector-shaped Computer Codes (NCAR TN-140+STR; Boulder: NCAR)

1980, Appl. Opt., 19, 1505

Wyatt, M. C., \& Dent, W. R. F. 2002, MNRAS, 334, 589

Wyatt, M. C., Dermott, S. F., Telesco, C. M., Fisher, R. S., Grogan, K., Holmes, E. K., \& Piña, R. K. 1999, ApJ, 527, 918

Zuckerman, B., \& Becklin, E. E. 1993, ApJ, 414, 793 